\title{
Methodologische Überlegungen zum Gestus literarischen Schreibens bei Florjan Lipuš
}

\author{
Dominik SRIENC \\ University of Klagenfurt (Austria)
}

\begin{abstract}
The topic of my research is the multilingual literary work of the Carinthian Slovene author Florjan Lipuš. My paper examines his work from a productional aesthetics point of view within a multilingual literary practice, using the example of the novel Bostjanov let (Bostjans Flug). For the first time, his literary estate, acquired by the Robert-Musil-Institute for Literary Research / Archives of Carinthian literature, is made accessible. It consists of all phases of the genetic history of his literary works, through the stages of his life cycle (notes, sketches, drawings, pencil shavings, manuscripts). Previous studies investigating the writing process lack the aspect of literary multilingualism. From the perspective of the (multilingual) writing process, the literature of Florjan Lipuš thus presents itself in a new light. Writing is not just the act of writing itself but consists of different moments. It is also a cultural process with political and multilingual implications. Florjan Lipuš has
\end{abstract}

received numerous prestigious awards (among them in 2018 the Grand Austrian State Prize for Literature) and his work was translated into various languages. My article, based on the research for my dissertation, addresses the question of how Lipuš writing process and his own relation to his work present itself in his literary estate. My article expands the range of the "gesture of literary writing" (Grésillon) by specifically applying it to the crucial social, political and historical tensions of the literary practice of Carinthian Slovenes. I argue that understanding the writing process is crucial to understanding the specific multilingual poetics of an author.

Keywords: Florjan Lipuš, Schreibprozessforschung, Boštjans Flug, Zweisprachige literarische Praxis, Literatur der Kärntner Slowenen, critique génetique, Literarische Mehrsprachigkeit

(c) by the author; dominik.srienc@aau.at

Colloquium: New Philologies, Volume 5, Issue 2 (2020)

doi: 10.23963/cnp.2020.5.2.8

Stable URL: https://colloquium.aau.at/index.php/Colloquium/article/view/139

This work is licensed under a Creative Commons Attribution 4.0 International License (CC BY 4.0). 


\section{Im „Wörterholz“. Zum Gestus des literarischen Schreibens bei Florjan Lipuš}

Die folgenden Überlegungen speisen sich aus offenen Fragen des zwischen 2016 und 2018 an der Karl-Franzens-Universität Graz durchgeführten FWF-Forschungsprojekts Die zweisprachige literarische Praxis der Kärntner Slowenen nach der Einstellung des mladje (1991) und ibre Position im überregionalen literarischen Interaktionsraum und ersten Ergebnissen meiner sich in Arbeit befindlichen Dissertation mit dem Titel Im „Wörterholz“. Zum Gestus literarischen Schreibens bei Florjan Lipuš, die von. Univ.-Prof. ${ }^{\text {in }}$ Dr. ${ }^{\text {in }}$ Anke Bosse (Universität Klagenfurt) und Univ.-Prof. Dr. Andrej Leben (Universität Graz) betreut wird. Mein Dissertationsvorhaben hat zum Ziel, neue literaturwissenschaftliche Zugänge zum Werk des Kärntner slowenischen Schriftstellers Florjan Lipuš zu schaffen. Es analysiert dieses Werk erstmals aus produktionsästhetischer Perspektive und erkundet die spezifische Arbeitsweise des Autors. Exemplarisch werden die Möglichkeiten einer textgenetischen Analyse der Werkmaterialien erprobt, die auf die mehrsprachige literarische Praxis und den Gestus literarischen Schreibens bei Lipuš ausgerichtet ist. Besonderere Berücksichtigung findet dabei Boštjanov let (2003) / Boštjans Flug (2005).

\section{Ausgangspunkte}

Als Florjan Lipuš Roman Zmote dijaka Tjaža 1981 in der deutschen Übersetzung von Peter Handke und Helga Mračnikar unter dem Titel Der Zögling Tjaž im ResidenzVerlag erschien, feierte der Autor aus dem Stand internationale Erfolge im deutschsprachigen und französischen Raum. Die Übersetzung leitete durch den damals bereits international bekannten Autor Handke eine „, noch nie dagewesene Blüte der slowenischen Literatur in Kärnten“ ein; doch die „Affirmation dieser Literatur zwischen zwei Nationalliteraturen“ kam, so Fabjan Hafner $(2009,18)$, zuerst von deutscher Seite und dann erst von slowenischer. Ungeachtet dieser Anerkennung und der Tatsache, dass Florjan Lipuš ein bedeutender Autor ist, gibt es bisher kaum größere wissenschaftliche Forschungsarbeiten zu seinem Werk. ${ }^{1}$ Eine Ausnahme stellen der 1998 von Johann Strutz herausgegebene Band Proflle der neueren slowenischen Literatur in Kärnten sowie der 2000 erschienene Sammelband Lipušlesen (Amann \& Strutz 2000) dar. Darin wird das Schaffen

1 Dies liegt vor allem daran, dass die nicht-deutschsprachige Literatur aus Österreich gerade in breit rezipierten Standardwerken zur österreichischen Literaturgeschichte nicht oder kaum behandelt wird, da sie unausgesprochen nicht zur österreichischen Literatur gezählt wird. Beispielhaft sei auf Wendelin Schmidt-Dengler verwiesen, der in seinen Bruchlinien. Vorlesungen zur österreichischen Literatur 1945 bis 1990 Florjan Lipuš in einem einzigen Satz erwähnt, nur um sich im nächsten Satz wieder seinem Übersetzer ins Deutsche, Peter Handke, zu widmen. (Schmidt-Dengler, 2010, 492). 
von Florjan Lipuš unter verschiedenen Blickwinkeln beleuchtet. In den fast zwanzig Jahren seither wurde die Forschung zur anhaltenden literarischen Produktion von Florjan Lipuš nicht systematisch erfasst und kaum aktualisiert. Dabei hat sich in genau diesen zwanzig Jahren der Kontext dieser Produktion, die literarische Praxis der Kärntner Slowenen und deren Wahrnehmung, grundlegend geändert. ${ }^{2}$ Das Schreiben von Kärntner Slowenen, ob in deutscher oder slowenischer Sprache, ist deutlich öffentlicher geworden, erfährt eine überregionale, sowohl in den slowenischsprachigen wie den deutschsprachigen Raum reichende Resonanz. ${ }^{3}$ Symptomatisch dafür ist, dass Florjan Lipuš seit 2000 mehrere bedeutende Preise erhielt, unter anderem den wichtigsten Preis für Literatur in Slowenien, den Prešeren-Preis (2004), oder den Großen Österreichischen Staatspreis für Literatur (2018) ${ }^{4}$. Das Buch Boštjanov let (2003) / Boštjans Flug (2005) wurde von Peter Handke (2017) zu „Weltliteratur!“ geadelt. In den 2010er Jahren ist Lipuš im österreichischen Literaturkanon angekommen und in einschlägigen Anthologien präsent: So ist der Tjaž-Roman in den von Klaus Kastberger (2013) herausgegebenen Grundbüchern der österreichischen Literatur seit 1945 ebenso vertreten wie in der von Bernhard Fetz herausgegebenen Reihe Österreichs Eigensinn.

Der größte Teil von Lipuš‘ Werk wurde dank Peter Handke, Helga Mračnikar, Fabjan Hafner und Johann Strutz aus dem Slowenischen ins Deutsche übersetzt; aufgrund eines Rechtsstreits zwischen dem Autor und seinem ehemaligen Verlag, dem Wieser Verlag, sind nicht alle deutschsprachigen Übersetzungen im Handel verfügbar (vgl. Klauhs 2015, 23). Mittlerweile mag der Erfolg für Lipuš groß sein, doch die Forschung zum ausnahmslos auf Slowenisch schreibenden Autor ist immer noch sehr übersichtlich und bewegt sich im Rahmen desselben Deutungshorizonts, der sich entlang einer überschaubaren Reihe wiederkehrender Themen und Motive erstellt: Autobiographie, Gesellschaftskritik, Zeitgeschichte, Identität, Sexualität und Sprache.

Gemeinsam ist diesen Interpretationsansätzen, dass sie auf produktionsästhetische Fragestellungen und auf Fragen nach der spezifischen Schreibweise des Autors nicht eingehen. So fehlt es nicht nur an umfassenderen, systematischen Untersuchungen zu Lipuš, vielmehr ist bislang auch keine einzige umfangreichere textgenetische Studie vorhanden. Der Forschungsartikel von Jože Blajs Das Tjaž-Projekt. Eine textgenetische Studie bezieht sich ausschließlich auf die abgedruckten Teilveröffentlichungen des Romans Zmote dijaka Tjaža / Der Zögling Tjaž in der Literaturzeitschrift mladje, ohne jedoch den literari-

\footnotetext{
2 In meiner Mitarbeit am FWF-Projekt „Die zweisprachige literarische Praxis der Kärntner Slowenen nach der Einstellung des mladje (1991) und ihre Position im überregionalen literarischen Interaktionsraum“ (Projektdauer: 2016-2018) wurde diesbezüglich eine erste Bestandsaufnahme gemacht.

3 Man denke etwa an den internationalen Erfolg von Maja Haderlap mit dem Roman Engel des Vergessens.

4 Der Preis war Lipuš 2017 mit der Begründung versagt geblieben, er schreibe nicht Deutsch. Zur ,peinlichen Affäre" rund um die Nicht-Verleihung des Staatspreises siehe Jung (2017).
} 
schen Vorlass zu berücksichtigen. Da die bisherige Forschung zu Lipuš Werk weder dessen Entstehung noch die Werkmaterialien berücksichtigte und den Gang ins Archiv verabsäumte, stellt sich mein Dissertationsvorhaben einem dringlichen Forschungsdesiderat. ${ }^{5}$ Zusätzlich profitiert mein Vorhaben davon, dass der zweite Teil des Lipuš-Vorlasses erst 2017 vom Land Kärnten angekauft, dem Musil-Institut/Kärntner Literaturarchiv als Dauerleihgabe übergeben wurde und seiner wissenschaftlichen Auswertung harrt. ${ }^{6}$

\section{Gestus des (mehrsprachigen) literarischen Schreibens}

Während es das Phänomen des Schreibens seit der Erfindung der Schrift im 4. Jahrtausend v. Chr. gibt, beschäftigten sich die literatur- und kulturwissenschaftlichen Disziplinen mit dem Thema ,Schreibprozesse vermehrt erst in den letzten Jahrzehnten. Von entscheidender Bedeutung ist dabei die von Louis Hay in den 1970er Jahren begründete und von Almuth Grésillon u.a. konsequent weitergeführte critique génétique. Diese ist an "generellen epistemologischen Fragestellungen [...], nämlich der Erforschung von Schaffens- und Erkenntnisprozessen“ (Grésillon 1999, 49) interessiert, welche sich am Archivmaterial ablesen lassen. Die critique génétique weitet den Werkbegriff aus und konzentriert sich auf Schreibprozesse, indem sie einzelne Entwürfe, Skizzen und Textfassungen als eigenständige Texte gegenüber der endgültigen Druckfassung eines Werks anerkennt. Um den Entstehungsprozess und die Arbeit am Text selbst zu rekonstruieren und zu interpretieren, nimmt sie alles, was als Teil der Textarbeit fassbar ist, in das sog. „dossier génétique“ auf. Ausgehend von einem dynamischen Literatur- und Textbegriff beschreibt Grésillon die Methodik der critique génétique als „Untersuchungen zu Form und Verlauf des Schreibens“, als Ausbildung von „Hypothesen über Schreibprozesse“ mit dem „Ziel: Literatur als ein Tun, eine Handlung, eine Bewegung“ zu erforschen und darzustellen (Grésillon 1999, 15). Neben der eigentlichen „Schreib-Zeit“, in der die Schreibprozesse stattfinden, sei auch die Zeitgeschichte von zentraler Bedeutung, in die sich der „Gestus literarischen Schreibens“ (Grésillon 1999, 49) einordne. Für den Gestus literarischen Schreibens spielten daher kulturpolitische und poetologische Faktoren ebenso eine Rolle wie das kulturelle bzw. literarische Erbe und der Kanon (Grésillon 1999, 49). Der Begriff des, Gestus' umfasst aber noch mehr, nämlich einerseits die konkreten körperlichinstrumentellen Handlungen der Geste und Gestik des Schreibens, die die je nach Autor

\footnotetext{
5 Eva Schörkhuber wählt in ihrer Dissertation „Zugänge zu einem Archiv der Literatur - entlang den Lektüren von Maja Haderlaps Engel des Vergessens, Bogdan Bogdanović' Die grüne Schachtel. Buch der Träume und Elfriede Jelineks Winterreise" (2017) einen dezidiert kulturwissenschaftlichen Ansatz, um drei Texte als Archive zu lesen, ohne jedoch den Gang ins Archiv als Institution zu wagen.

6 Den Ankauf haben die Leiterin des Musil-Instituts / Kärntner Literaturarchivs, Anke Bosse, und ich initiiert.
} 
individuelle ,Schreibszene und die Produktivität des Schreibens bestimmen; und andererseits schließt, Gestus' auch den Ausdruck einer gesellschaftlichen Handlung, den Habitus, ein. ${ }^{7}$ Daher ist der Versuch Stephan Porombkas (Porombka 2006) naheliegend, die critique génetique mit Pierre Bourdieus Habitus-Theorie und seiner Theorie des literarischen Felds ins Verhältnis zu setzen. So ließen sich mit den Methoden der critique génetique neben Schreibprozessen und Textgenese auch deren Vernetzung mit dem Literaturbetrieb und dessen verschiedenen Akteuren als am Entstehungsprozess Beteiligten untersuchen.

Was Schreiben eigentlich sei, versucht die literaturwissenschaftliche Schreib- und Schreibprozessforschung zu ergründen, zu der das von Martin Stingelin, Sandro Zanetti und Davide Giuriato geführte Forschungsprojekt Zur Genealogie des Schreibens. Die Literaturgeschichte der Schreibszene von der Frühen Neuzeit bis zur Gegenwart samt zugehöriger Buchreihe wesentliche Beiträge vorgelegt hat. Das Projekt zielt theoretisch wie praktisch auf eine „systematische und historische Erforschung literarischen Schreibens [...] im Spiegel medientechnikhistorischer Umwälzungen“ (Morgenroth 2012,7). In diesem Sinne erweitert es die „Verständnismöglichkeiten literarischen Schreibens“ (NuttKofoth 2006, 230) Angelpunkt ist hier Rüdiger Campes (Campe 1991) kulturwissenschaftliches Konzept der „Schreibszene“, die den sprachlich-gestischen Ausdruckswert des Schreibens bezeichnet. Nach Campe funktionieren Schreiben und der damit verbundene Schreibakt als je nach Autor und Autorin individuelles und veränderliches Zusammenspiel dreier heterogener Faktoren: Zum Schreiben bedarf es des Wissens und Könnens, mit Sprache umzugehen (,Sprachlichkeit'), und es bedarf bestimmter Schreibinstrumente und -materialien (,Instrumentalität'), mit denen die Schreibenden umgehen können müssen, gestisch und körperlich (,Körperlichkeit'). Diesen Ansatz Campes erweitert Stingelin dadurch, dass er alle drei Faktoren als „Quelle möglicher Widerstände“ darstellt; da, „wo sich dieses Ensemble in seiner Heterogenität und Nicht-Stabilität an sich selbst aufzuhalten beginnt, thematisiert, problematisiert und reflektiert," entsteht nach Stingelin $(2004,15)$ die ,Schreib-Szene‘ (mit Bindestrich). Anke Bosse erweitert Campes drei Kriterien der ,Sprachlichkeit', ,Instrumentalität‘ und ,Körperlichkeit‘ des Schreibens um ein viertes, die „Theatralität des Schreibens“; diese äußere sich im Schreiben als „Performance, die sich selbst inszeniert" und als ein „In-Szene-Setzen“ der „Gelingensbedingungen“ des Schreibens (Bosse 2019, 2).

Sandro Zanetti $(2015,13)$ versteht „Schreiben als Kulturtechnik“, es sei „sowohl kulturell bedingt als auch kulturkonstituierend“, denn ein „kulturelles Gedächtnis, ja Kultur überhaupt kann sich ohne Praktiken der Aufzeichnung nicht längerfristig etablieren“.

\footnotetext{
7 „Gestus“ auf Duden online. URL: https://www.duden.de/node/689347/revisions/2002227/view (Zugriff am 27.3.3019).
} 
Nach Zanetti $(2015,7)$ verweisen Schreibakte immer schon über den puren „Aufzeichnungsakt“ hinaus, weil in ihnen „Erinnerungen, Erfahrungen und Wissensbestände produziert, artikuliert und organisiert werden“. Schreibakte hinterlassen Spuren, das Geschriebene wird archiviert. Neben dieser "Tendenz zur Selbstarchivierung" sei es auch notwendig, dass das Geschriebene aufbewahrt werde, denn Schriftstücke durchlaufen „Transformationsprozesse, Übersetzungsprozesse, Vervielfältigungen. [...] Sie werden funktionalisiert, selektioniert, publik gemacht oder geheimgehalten [sic]. Sie gelten als erinnerungswürdig, werden vergessen oder vernichtet“"(Zanetti 2012,31).

Mit dem zwölften Band der Reihe Zur Genealogie des Schreibens werden die Konzepte der Schreibszene und der Schreib-Szene um eine politische Dimension erweitert. Die Schreibszene, die Schreib-Szene sowie das Moment des Widerstands ermöglichten es,

die philologischen und schreibmaterialen Situationen des Schreibens und des Schreibenden als auch ihre sozialen Begleitumstände in ein praxeologisches Modell zu integrieren, mit dem nun aus einer produktionsästhetischen Perspektive heraus alle Phasen des literarischen Arbeitsprozesses, vom Einfall bis zum Druck, einzeln oder in ihrem Zusammenspiel auf ihre politische Dimension hin untersucht werden können. (Morgenroth et. al. 2008, 9)

Zwei weitere wichtige Perspektiven lässt die Schreibprozessforschung bisher weitgehend außer Acht: Was die „Übersetzungsszene“ (Stingelin 2006) sei, hat sie nur gestreift, und Phänomene literarischer Mehrsprachigkeit hat sie bislang nicht berücksichtigt. Ihre theoretischen wie praktischen Fragestellungen kennzeichnen sich durch eine merkwürdige Abstinenz gegenüber literarischer Zwei- bzw. Mehrsprachigkeit. Dies ist umso erstaunlicher, als diese Fragestellung bereits seit den 1980er Jahren mit dem immer prominenteren Auftreten von zwei- und mehrsprachigen Autoren und Autorinnen in den Fokus der Literaturwissenschaft gerückt ist. Denn (literarische) Mehrsprachigkeit ermöglicht den „Zugang zu Phänomenen sprachlicher, kultureller und sozialer Differenz", ist eine „Herausforderung für die philologischen Instrumente“ der Textanalyse und impliziert die „Möglichkeit der Überwindung nationalphilologischer Betrachtungsweisen“ (Dembeck und Parr 2017, 9). Hier ist ein weiteres Forschungsdesiderat zu erkennen: Es fehlt bislang ein produktionsästhetischer Ansatz, mit dem genau jene Schreibprozesse beschrieben werden können, die über literarische Mehrsprachigkeit und Übersetzungsprozesse in Gang gesetzt, von ihnen gerahmt und gesteuert werden. Auch hier soll meine Dissertation eine Forschungslücke schließen. 


\section{Lipuš und die „Zweisprachige literarische Praxis der Kärntner Slowenen“}

Um Phänomene von literarischer Mehrsprachigkeit in Beziehung zu Schreibprozessen zu setzen, bietet sich gerade die „polyphone, transkulturelle écriture“ der „Alpen-AdriaRegion“" (Strutz 2010, 178) als Forschungsfeld an. Besondere Bedeutung im literarischen Einzugsbereich dieser Region kommt der zwei- oder mehrsprachigen literarischen Produktion der Kärntner Slowenen zu. Basierend auf Ergebnissen des FWF-Forschungsprojekts Die zweisprachige literarische Praxis der Kärntner Slowenen nach der Einstellung des mladje (1991) und ihre Position im überregionalen literarischen Interaktionsraum erscheint 2021 die von Andreas Leben, Erwin Köstler, Felix Kohl und mir verfasste Monographie Überregional, mehrsprachig, vernetzt. Die Literatur der Kärntner SlowenInnen im Wandel. ${ }^{8}$ In dieser Monographie werden Aspekte literarischer Zwei- und Mehrsprachigkeit und die Interaktionen im überregionalen literarischen Interaktionsraum beforscht. Erste Ergebnisse zeigen, dass sich die Literatur der Kärntner Slowenen nach 1991 grundlegend gewandelt hat. Literatur wird besonders von den um 1960 oder später geborenen Autorinnen und Autoren vielfach auf Slowenisch und/oder Deutsch verfasst, Publikations- und Rezeptionsmöglichkeiten außerhalb der klassischen Minderheitenstrukturen genutzt. Sprache (Slowenisch) und Territorium (Kärnten) reichen nicht mehr aus, um die Literatur der Kärntner Slowenen adäquat zu beschreiben. ${ }^{9}$

Vor diesem Hintergrund ist das literarische Schaffen des 1937 in Lobnig/Lobnik oberhalb von Eisenkappel/Železna Kapla geborenen Autors Florjan Lipuš zu sehen. Es ist in mehrfacher Hinsicht ungewöhnlich. Lipuš schreibt von Anfang an seine Theaterstücke, Romane und Essays auf Slowenisch. So überträgt er ihr, der slowenischen Sprache, die Präfiguration und Regie seines Schreibens und seiner ästhetischen Weltentwürfe. Das Schreiben auf Slowenisch ist bei Lipuš von Anfang an auch als konsequente Schreibpolitik, als ideologische Position und als existenzieller Akt des Widerstands zu verstehen: „Daß ich auf Slowenisch schreibe und dabei bleibe, macht meine Literatur genug engagiert, genug politisch“ (Amann und Strutz 2000, 28), sagt Lipuš, der in seinen Texten immer wieder auf politische und gesellschaftliche Fragen fokussiert. Schon in seiner Schulzeit im Internat Tanzenberg vertrat Lipuš eine Protesthaltung gegen die ideologische Vereinnahmung der Literatur durch ein artistisches Sprachkonzept. Dieses kulminiert, so Erich Prunč (Prunč 2013, 144) in folgendem Statement: „Die slowenische Literatur in Kärnten als Literatur der Erniedrigten und Beleidigten ist allein dadurch enga-

\footnotetext{
8 Kohl Felix, Erwin Köstler, Andreas Leben und Dominik Srienc. 2021. Überregional, mehrsprachig, vernetzt. Die Literatur der Kärntner SlowenInnen im Wandel. Wien: Praesens.

9 Allgemein dazu Leben (2019), spezifisch Srienc (2019).
} 
giert, dass sie ist." Als Mitbegründer und Herausgeber der Zeitschrift mladje hat Lipuš in den 1960er Jahren bewusst mit den traditionalistischen und kulturkonservativen Vorläufern der Nachkriegszeit gebrochen. Die Autoren rund um diese Zeitschrift strebten, von Lipuš gefördert und unterstützt, nach gesellschaftlicher und literarischer Emanzipation.

Die historischen Rahmenbedingungen und Voraussetzungen seines literarischen Erzählund Schreibprogramms sind auch vor der Folie der Geschichte der Kärntner Slowenen zu verstehen, die, wie Johann Strutz (Strutz 1998, 79) feststellt, als „regionaler Prätext des literarischen Werks“ von Florjan Lipuš fungiert. Zwei tragische Erfahrungen durchziehen alle Texte von Lipuš und treiben sie an: die Verhaftung der Mutter, die vor den Augen der beiden kindlichen Söhne von SS-Schergen abgeführt wird und aus dem KZ Ravensbrück nicht zurückkehrt, und der Tod der Großmutter, der das Kind Lipuš und seinen Bruder in völlige, existentiell bedrohliche Verlassenheit stieß. Die beiden Buben wurden nur durch Zufall entdeckt und gerettet. Diese zwei traumatisierenden Kindheitserlebnisse prägen den Eigensinn der Texte Lipušs, die, so Fabjan Hafner (Hafner 2016), von „hohem ästhetischem Ehrgeiz und vehementem Engagement“ geprägt sind.

Wer mit dem Begriff, Literarische Mehrsprachigkeit' arbeitet, tut dies vor dem Hintergrund einer beinahe unüberschaubaren Zahl an Definitionen. Im Gegensatz ließe sich, so Till Dembeck und Rolf Parr in ihrer Einleitung zum Standardwerk literaturwissenschaftlicher Mehrsprachigkeitsforschung Literatur und Mehrsprachigkeit (2017, 10), zudem definitorisch nicht sagen, was ein einsprachiger Text eigentlich sei. Das terminologische und definitorische Dilemma rund um den Komplex ,Literarische Mehrsprachigkeit' kann in diesem Artikel nicht beleuchtet werden, es sei hier lediglich auf die seit den 1990er Jahren vermehrten Untersuchungen verwiesen, die den Gegenstand stärker in den Blick gerückt haben. ${ }^{10}$ Literarische Mehrsprachigkeit lässt sich, so sowohl in ihren außertextuellen kulturellen, sozialen Rahmenbedingungen, aber auch auf der Textebene, in sprachlichen Ausprägungen verstehen. Rolf Dembeck und Till Parr (2017) stellen ein brauchbares Instrumentarium für die Analyse mehrsprachiger Texte bereit. Zu den „Basisverfahren literarischer Mehrsprachigkeit“ (Dembeck und Parr 2017, 12) zählen Sprachwechsel, Sprachmischung, Mehrsprachigkeit in der Figurenrede, anderssprachige Zitation, Mehrschriftlichkeit und Übersetzung. Alle Basisverfahren lassen sich in manifester und latenter Form beobachten. Für Giulia Radaelli ist die Antwort auf die Frage, was Literarische Mehrsprachigkeit sei, innerhalb der Texte und nicht im Kontext zu suchen: „Literarische Mehrsprachigkeit, mehrsprachige Literatur sind mehrsprachige literarische Texte“ (Radaelli 2014, 157). Letzterer Definition nach, auf immanenter Textebene ließe sich der laut mehrmaliger Selbstauskunft explizit ,einsprachige, da am

${ }^{10}$ Zur Forschungsgeschichte siehe Helmich (2016, 22-46), sowie eine aktuelle Auswahlbibliographie bei Dembeck und Parr $(2017,347-362)$. 
Slowenischen als Literatursprache festhaltende Autor Florjan Lipuš als mehrsprachiger Autor bezeichnen. Um die literarische Produktion der Kärntner Slowenen innerhalb des Arbeitsfeldes, Literarische Mehrsprachigkeit ' zu verorten, sind aber auch textexterne Faktoren literarischer Mehrsprachigkeit zu berücksichtigen, um dem, Gestus' mehrsprachigen, literarischen Schreibens gerecht zu werden. Im Kontext der politisch wie ideologisch überfrachteten Frage nach dem Slowenischen oder dem Deutschen als Literatursprache ist dieser besondere einsprachige Gestus in der gegenwärtigen literarischen Praxis der Kärntner Slowenen zu hinterfragen. Feststeht, dass Lipuš als einer der Hauptakteure innerhalb der literarischen Praxis der Kärntner Slowenen, am Slowenischen als Literatursprache festhält, seine Texte aber gekennzeichnet sind von einer innersprachlichen Mehrsprachigkeit, die literarisch belegbar ist in der der „Ausdrucksfülle“, der „stilistischen Wandlungsfähigkeit“ bis hin zur „experimentelle[n] Sprachäußerung“ (Borovnik 2019, 129). Fragt man nach der „Mehrsprachigkeit des Gesamtwerks“ (Radaelli 2014, 161) bei Florjan Lipuš, wird der einsprachige Gestus des Schreibens des Autors relativiert. Einige wenige der literarischen wie publizistischen Texte von Lipuš wurden auf Deutsch verfasst, zudem kommt bei Lipuš, dessen Werk nahezu zur Gänze auch in deutscher Übersetzung vorliegt, auch die Frage nach der Übersetzung als Form literarischer Mehrsprachigkeit ins Spiel.

\section{Gang ins Archiv - Autopsie des Materials}

Welche Institutionen sammeln Bestände mehrsprachiger Autoren? Gibt es überhaupt mehrsprachige Bestände in den Literaturarchiven? Wer bearbeitet diese? In welcher Sprache, welchen Sprachen wird den Beständen begegnet? Ein Blick in die „Archive der Kärntner slowenischen Literatur" (Srienc 2020) zeigt, dass nur sehr wenige Literaturarchive in Österreich methodisch Bestände Kärntner slowenischer Autoren sammeln, nur wenige Kärntner slowenische Autoren sind bislang archiviert, Bestände und Sammlungen zu slowenischer Literatur befinden sich etwa im Robert-Musil-Institut für Literaturforschung / Kärntner Literaturarchiv (RMI / KLA), aber vereinzelt auch in Literaturarchiven in Österreich, Deutschland und Slowenien (vgl. Srienc 2020). Der Vorlass von Florjan Lipuš befindet sich seit 1997 im RMI / KLA, das Archiv von Lipuš ist ein Papierarchiv. Der zweite Teil des Vorlasses wurde 2017 vom Land Kärnten angekauft und dem MusilInstitut / Kärntner Literaturarchiv als Dauerleihgabe übergeben. Zum zweiten Teil des Vorlasses zählt Material zu Werken, die um und nach 2000 erschienen sind, darunter etwa Boštjanov let / Boštjans Flug. Das Werkmaterial umfasst u.a. Schulhefte aus der Tanzenberger Zeit, die für das Schreiben von Lipuš charakteristischen Bleistiftmanuskripte, Reinschriften oder Übersetzungstyposkripte. 


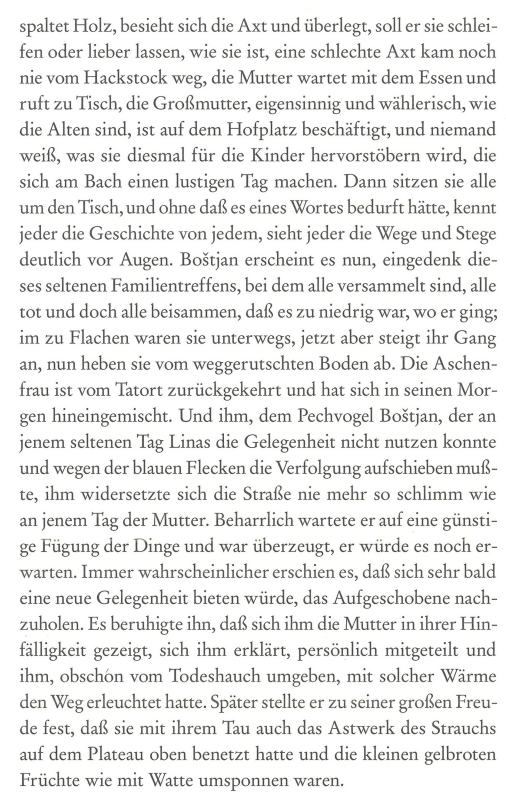

150

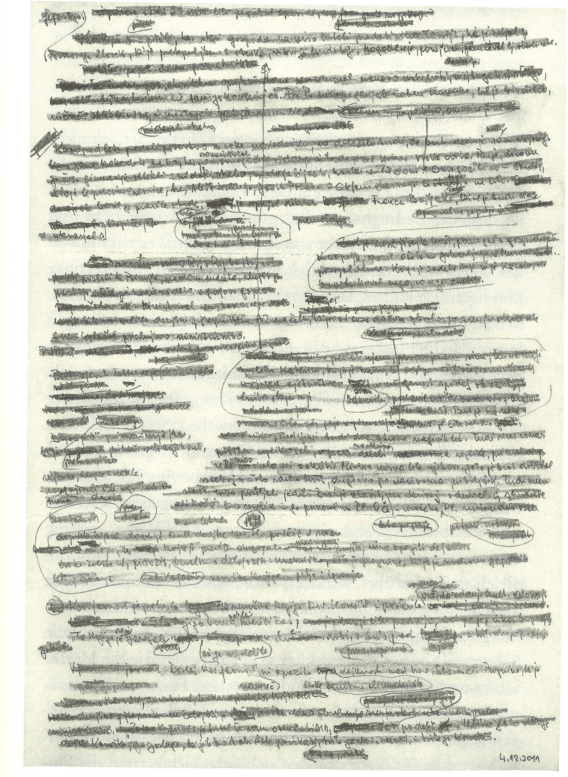

Abbildung 1: Text- und Manuskriptseite, abgedruckt in Florjan Lipuš: Boštjans Flug (2013).

Florjan Lipuš Schreiben ist von Anfang an ein konsequentes Schreiben mit dem Bleistift. Die Arbeit mit dem Bleistift ist entscheidende, notwendige Produktionsbedingung seines Schreibens: „Ich schreibe mit Bleistift, soweit ich mich zurückerinnern kann, Literarisches nur mit dem Bleistift, sonstiges mit allerlei Schreibbarem. Und ich schreibe bis zum letzten Schriftzug der Mine.“ (Waldner-Petutschnig 2018). Doch schreibt Lipuš die Bleistifte nicht nur mit Hilfe einer Verlängerung zu Stummeln herunter, auch das beschriebene Material, Schmierblätter und Schulhefte, bezeugen eine Sparsamkeit und Entsagung, die aus der Kindheit herrührt.

In seinem kurzen Aufsatz Im Bleistiftgebiet widmet sich Fabjan Hafner nicht nur Lipuš Schatulle mit Bleistiftstummeln - die nicht weggeworfen, sondern immer noch für potenzielle Schreibarbeiten aufgehoben werden -, sondern auch dem äußeren Erscheinungsbild und der Topographie der Bleistiftmanuskripte. Ihr Schriftbild vergleicht er mit „Landschaften oder Landkarten“, mit „Inseln oder Oasen“ (Hafner 2015, 211). Lipuš benutzt sehr harte Bleistifte zum Schreiben, seine Schrift ist winzig und doch zugleich erstaunlich leserlich; weiche Bleistifte benutzt er, um auf seinen Schmierblättern jene Zeilen mit einer Art Schleier zu überziehen, die er auf einem anderen Blatt ins Reine geschrieben hat. Der Bleistiftschleier zeigt einerseits an, was überholt, da weiterverarbei- 
tet ist, und andererseits ein immer noch erkennbares Geschriebenes, das potenziell wiederbelebt werden kann. Um dieses Reservoir zu erhalten, hat Lipuš seine Schmierblätter sorgfältig aufgehoben. Auf diesen Schmierblättern arbeitet er an Textkernen, die er anreichert, erweitert, zu einem größeren Ganzen zusammenfügt und dann mit Bleistift ins Reine schreibt oder - später - am PC abtippt. Dann werden die Texte ausgedruckt und erneut handschriftlich bearbeitet.

An den Schmierblättern ist außerdem interessant, dass sie Indizien über die Zeit der Niederschrift enthalten. Sobald Lipuš alle Textpassagen eines Blattes in die Reinschrift übertragen hat, belegt er sie nicht nur mit seinem charakteristischen Bleistiftschleier, sondern setzt unten auf die Seite auch ein Tagesdatum. Die einzelnen, getilgten Passagen werden nach dem Übertragen in die Reinschrift datiert. Lipuš betont die Produktivität durch den skripturalen Akt der Streichung. Dafür sorgen auch die peniblen Datierungen der jeweiligen Tagesleistungen. Der Bleistiftschleier tritt in den Dienst einer zielorientierten Textproduktion. Zudem entwirft Lipuš Figurennamen und erprobt eventuelle Arbeitstitel in den Schmierblättern, um diese in der Reinschrift und in den Computerausdrucken noch einmal zu ändern. Lipuš selbst nennt das spezifische Erscheinungsbild seiner eigenen Schrift „besedna suhljad“, von Johann Strutz übersetzt mit „Wörterholz“ (Lipuš 2017, 14).

\section{Stellenwert für die Forschung}

Das Hauptanliegen meiner Dissertation ist es, der Frage nachzugehen, wie das Schreiben von Florjan Lipuš, ob einsprachig oder mehrsprachig im Werkmaterial zu Boštjanov let / Boštjans Flug in Erscheinung tritt. Noch vor dem Gang ins Archiv ist es möglich, sich in der Dissertation einen Überblick über die Beschaffenheit und Aussagekraft des Werkmaterials zu verschaffen, dabei stellen sich unmittelbar Fragen nach den (mehrsprachigen) Schreibspuren im Material, nach der Dynamik des Schreibprozesses. Paradigmen der Schreibprozessforschung und textgenetischen Kritik werden mit aktuellen Fragen der literarischen Mehrsprachigkeit und der zweisprachigen literarischen Praxis verflochten. Neben textgenetischen Fragestellungen gilt das wissenschaftliche Interesse auch der Genese gesellschaftlich-politischer Diskurse, die für die Literatur der Kärntner Slowenen von zentraler Bedeutung sind. Welches sind die spezifischen Rahmenbedingungen, in denen der, Gestus literarischen Schreibens ‘ bei Florjan Lipuš vollzogen wird? Was sind seine ästhetischen, politischen, sozialen Voraussetzungen? Welche Rolle spielen Mehr- und Einsprachigkeit in und für sein Schreiben? In welcher Hinsicht enthalten die Schreib-Szenen bei Lipuš Dimensionen des Politischen? Wie hat sich das Schreiben der Kärntner Slowenen historisch transformiert, wo liegen ihre literarischen Archive und in 
welcher kulturellen Formation? Dem Dissertationsvorhaben liegt die Überzeugung zugrunde, dass über einen bewusst materialästhetischen Zugang neue Fragen an das Schreiben und die literarische Praxis von Florjan Lipuš gestellt werden können. Damit sind die Ziele dieser komparatistischen Arbeit umrissen. Sie wird die erste umfassende Untersuchung sein, die sich Florjan Lipuš Schreibprozessen widmet, inklusive ihrer politischen und (mehr-)sprachlichen Bedingungen.

\section{Literatur}

Amann, Klaus und Johann Strutz. 2000. „Florjan Lipuš. Kleines Porträt mit Hintergrund.“ In Lipušlesen. Texte und Materialien zu Florjan Lipuš, hrsg. von Klaus Amann und Johann Strutz, 9-28. Klagenfurt, Wien, Ljubljana, Sarajevo: Wieser.

Blajs, Jože. 2000. „Das Tjaž-Projekt. Eine textgenetische Untersuchung.“ In Lipušlesen. Texte und Materialien zu Florjan Lipuš, hrsg. von Klaus Amann und Johann Strutz, 59-82. Klagenfurt, Wien, Ljubljana, Sarajevo: Wieser.

Borovnik, Silvija. 2019. „Form und Bedeutung des Slowenischen in der Literatur Florjan Lipušc“ In Literarische Mehrsprachigkeit im österreichischen und slowenischen Kontext, hrsg. von Alenka Koron und Andreas Leben, 127-140. Tübingen: Narr Francke.

Bosse, Anke. 2019. „Die Wortmaschine ... wird jetzt in Betrieb genommen“. Schreibszene, Überlappungszone und Schreib-Szene bei Josef Winkler." In Textgenese in der digitalen Edition, hrsg. von Anke Bosse und Walter Fanta, 1-15. Berlin, New York: de Gruyter (= Beihefte zu editio).

Campe, Rüdiger. 1991. „Die Schreibszene. Schreiben. In Paradoxien, Dissonanzen, Zusammenbrüche. Situationen offener Epistemologie, hrsg. von Hans Ulrich Gumbrecht und K. Ludwig Pfeiffer, 759-772. Frankfurt am Main: Suhrkamp.

Dembeck, Till und Rolf Parr. 2017. „Mehrsprachige Literatur. Zur Einleitung.“ In Literatur und Mehrsprachigkeit. Ein Handbuch, hrsg. von Till Dembeck und Rolf Parr, 9-14. Tübingen: Narr Francke Attempto Verlag.

Grésillon, Almuth. 1999. Literarische Handschriften. Einfübrung in die „critique génétique“. Bern, Wien: Lang.

Hafner, Fabjan. 2009. „Der „exemplarische Epiker“ der Kärntner SlowenInnen: Florjan Lipuš. In Und (k)ein Wort Deutsch ... Literaturen der Minderheiten und MigrantInnen in Österreich, hrsg. von Nicola Mitterer et. al., 133-150. Wien, Innsbruck: Studien-Verlag.

Hafner, Fabjan. 2015. „Florjan Lipuš. Im Bleistiftgebiet“ In Das Literaturmuseum. 101 Objekte und Geschichten, hrsg. von Bernhard Fetz, 211. Salzburg: Jung und Jung.

Handke, Peter. 2017. „Aufruhr und Liebe“. In Boštjans Flug, von Florjan Lipuš, 163-167. Berlin: Suhrkamp (=3. Auflage).

Helmich, Werner. 2016. Asthetik der Mehrsprachigkeit. Zum Sprachwechsel in der neueren romanischen und deutschen Literatur. Heidelberg: Winter (= Studia Romanica Band 196).

Jung, Jochen. 2017. „Peinliche Affäre rund um Österreichischen Staatspreis.“ Die Presse, 3. Jänner 2017. https://diepresse.com/home/meinung/gastkommentar/5149018/Peinliche-Affaererund-um-Oesterreichischen-Staatspreis. (Zugriff am 20.3.2020). 
Klauhs, Harald. 2015. „Haben wir bald einen Staatspreisträger ohne Hauptwerk?“. Die Presse, 9. Juli 2015. http://diepresse.com/home/kultur/literatur/4773543/Haben-wir-bald-einenStaatspreistraeger-ohne-Hauptwerk. (Zugriff am 18.2.2019).

Kohl Felix, Erwin Köstler, Andreas Leben und Dominik Srienc. 2021. Überregional, mehrsprachig, vernetzt. Die Literatur der Kärntner SlowenInnen im Wandel. Wien: Praesens. Im Druck.

Leben, Andreas. 2019. „Zum Modell und zum Begriffsfeld des überregionalen literarischen Interaktionsraums (ausgehend von der Literatur der Kärntner Slowen_innen).“ In Literarische Mehrsprachigkeit im österreichischen und slowenischen Kontext, hrsg. von Alenka Koron und Andreas Leben, 63-77. Tübingen: Narr Francke Attempto.

Lipuš, Florjan. 2013. Boštjans Flug. Übersetzt aus dem Slowenischen von Johann Strutz. Mit einem Nachwort von Peter Handke. Berlin: Suhrkamp.

Lipuš, Florjan. 2017. Seelenruhig. Übersetzt aus dem Slowenischen von Johann Strutz. Mit einem Nachwort von Fabjan Hafner. Salzburg: Jung und Jung.

Morgenroth, Claas, Martin Stingelin und Matthias Thiele. 2012. „Politisches Schreiben. Einleitung." In Die Schreibszene als politische Szene, hrsg. von Claas Morgenroth, Martin Stingelin und Matthias Thiele, 7-33. München: Fink (=Zur Genealogie des Schreibens Band 14).

Nutt-Kofoth, Rüdiger. 2006. „Rezension zu ,SCHREIBKUGEL IST EIN DING GLEICH MIR: VON EISEN“' Schreibszenen im Zeitalter der Typoskripte, hrsg. von Davide Giurato, Martin Stingelin und Sandro Zanetti. München: Wilhelm Fink 2005. (Zur Genealogie des Schreibens. 2), 311 S., Abb.“ In Editio 20. 229-232.

Porombka, Stephan. 2006. „Literaturbetriebskunde. Zur „genetischen Kritik“ kollektiver Kreativität." In Kollektive Kreativität, hrsg. von Stephan Porombka, 72-87. Tübingen: Francke.

Radaelli, Giulia. 2014. „Literarische Mehrsprachigkeit. Ein Beschreibungsmodell (und seine Grenzen) am Beispiel von Peter Waterhouses ,Das Klangtal““ In Philologie und Mehrsprachigkeit, hrsg. von Till Dembeck und Georg Mein, 157-179. Heidelberg: Winter-Verlag.

Schörkhuber, Eva. 2019. Akte (n) der Verwahrung. Zugänge zu einem Archiv der Literatur entlang exemplarischer Lektüren von Maja Haderlap, Bogdan Bogdanović und Elfriede Jelinek. Wien: Praesens.

Schmidt-Dengler, Wendelin. 2010. „Peter Handke: Die Wiederholung (1986)““ In Bruchlinien. Vorlesungen zur österreichischen Literatur 1945-1990, hrsg. von Wendelin Schmidt-Dengler, 484-502. St. Pölten / Salzburg: Residenz Verlag (=3. Korr. Auflage).

Srienc, Dominik. 2019. „Wie produziere ich als slowenischer Autor in Kärnten 2.0? Zum literarischen Selbstverständnis neuerer Kärntner slowenischer Literatur im Spannungsfeld zwischen Mehrsprachigkeit und Innovation." In Literarische Mehrsprachigkeit im österreichischen und slowenischen Kontext, hrsg. von Alenka Koron und Andreas Leben, 97-110. Tübingen: Narr Francke Attempto.

Srienc, Dominik. 2020. „Kleine Literaturen - kleine Archive? Zur Lesung und Sammlung Kärntner slowenischer Literatur." In Logiken der Sammlung. Das Archiv zwischen Strategie und Dynamik, hrsg. von Petra Maria Dallinger und Georg Hofer, 91-104. Berlin: De Gruyter.

Stingelin, Martin. 2006. „Schreiben (Raymond Roussel, Georges Perec) und Übersetzen. “In Prospero. (Band MMVI), hrsg. von Stingelin, Martin et. al., 41-55. (= Sonderheft Beiträge zum Studientag „Schreiben und Übersetzen“, Triest 10.5.2004). 
Strutz, Johann. 1998. Profile der neueren slowenischen Literatur in Kärnten. Klagenfurt/Celovec: Hermagoras Verlag/Mohorjeva založba (=2. Erweiterte Auflage).

Strutz, Johann. 2007. „Graben, Kehre und Schrift. Räume, Bewegungen und Koordinaten in der Schreibwelt von Florjan Lipuš." In Graben, Kehre, Schrift. Essays zu Florjan Lipuš, hrsg. Johann Strutz, Boris Paternu und Helmut Moysich, 7-14. Klagenfurt: Wieser.

Strutz, Johann. 2010. „Anstelle eines Nachworts: Dialog, Polyphonie und die Mehrsprachigkeit der Literatur." In Sprachlandschaften. Regionale Literaturwissenschaft im europäischen Kontext (kontext mosaik. Materialien zur kulturellen Diversität), hrsg. von Reinhard Kacianka et. al., 178-207. Klagenfurt/Celovec, Ljubljana/Laibach, Wien/Dunaj: Verlag Hermagoras/Mohorjeva založba.

Waldner-Petutschnig, Karin. 2018. „Vor lauten Menschen sollte man sich fürchten”. Interview mit Florjan Lipuš. Kleine Zeitung, 30. September 2018. 70-71.

Zanetti, Sandro. 2012. „Einleitung." In Schreiben als Kulturtechnik. Grundlagentexte, hrsg. von Sandro Zanetti, 7-34. Berlin: Suhrkamp (=2. Auflage 2015). 